\title{
La viticulture en crise à Laure-Minervois, Aude
}

Monique Barrué-Pastor

\section{Citer ce document / Cite this document :}

Barrué-Pastor Monique. La viticulture en crise à Laure-Minervois, Aude. In: Revue géographique des Pyrénées et du SudOuest, tome 50, fascicule 2, 1979. Paysannerie, exploitations agricoles. pp. 371-375;

doi : https://doi.org/10.3406/rgpso.1979.3605

https://www.persee.fr/doc/rgpso_0035-3221_1979_num_50_2_3605

Fichier pdf généré le 06/04/2018 
REVUE GÉOGRAPHIQUE DES PYRÉNÉES ET DU SUD-OUEST TOME 50, FASC. 2, pp. 371-378, Toulouse, 1979

\section{CHRONIQUE}

\section{LA VITICULTURE EN CRISE \\ A LAURE-MINERVOIS (AUDE)}

Plutôt que les luttes viticoles elles-mêmes, dont d'autres ont su parler avant nous, ce qui est au cœur de notre réflexion, c'est le pourquoi de ces luttes, de leur force et de leur violence, dans les formes diversifiées qu'elles ont prises, attestant une profonde hétérogénéité de vues et de pensée. Nous avons essayé de mettre en évidence les fondements de cette hétérogénéité en même temps que les raisons qui conduisent à la recherche d'une "unité viticole». Ce travail de reherche sur un vignoble et une société viticole que nous connaissions depuis longtemps poursuivait donc trois objectifs fondamentaux (1).

Analyser d'abord la nature profonde de la crise viticole; en rendre compte dans ce qu'elle recèle de fondements liés à la crise agraire que connaissent les pays où le capitalisme est parvenu à sa phase monopoliste. Cela passe par la mise en évidence de l'évolution des rapports entre les différents facteurs de production dans l'agriculture paysanne. Une partie de ce travail constitue donc une contribution à l'analyse de l'exploitation du travail paysan dans le système économique actuel; elle a conduit à l'étude de l'ensemble des structures sur lesquelles repose la production viticole: la terre, le travail et les structures d'exploitation, ainsi que les structures de production et de commercialisation.

Analyser ensuite la spécificité de la crise viticole: processus évolutifs, causes et conséquences des problèmes propres à la viticulture. On sait quelles sont les questions habituellement posées : la crise viticole est-elle la crise des vins de consommation courante ? La production est-elle excédentaire? Faut-il arracher le vignoble et par quoi peut-on le remplacer? Les coopératives viticoles sont-elles vouées à devenir des cathédrales vides? Il s'agit de réfuter les lieux communs sur le gros rouge; de réfuter aussi l'explication de la crise selon laquelle le vignoble méridional est largement responsable, sinon de la crise, du moins de ses malheurs, parce qu'il produit à gros rendement un vin de mauvaise qualité qu'il faut partiellement couper avec des vins italiens... ou mettre à la chaudière en

1. Monique Pastor-Barrué. Viticulteurs en crise à Laure-Minervois. Toulouse, 1978, Université de Toulouse-Le Mirail (Thèse de $3^{\circ}$ cycle, Géographie). 
vue de sa distillation. C'est l'étude des fondements de la crise viticole, et donc de son devenir par rapport au type de fonctionnement actuel, notamment en liaison avec l'évolution du Marché commun; c'est aussi l'étude de la validité des diverses mesures proposées et des possibilités.

Analyser enfin la société viticole. Qui est atteint par la crise? Qui sont et que sont les viticulteurs ? Il s'agit de saisir le processus de crise dans son mouvement et la diversité de ses effets selon les différentes composantes de la population. Le terrain d'observation est communal; mais la visée, ici, n'est nullement monographique; l'unité d'observation de base est l' " exploitation "; elle permet de déceler les fondements de cette hétérogénéité (économique, sociale et idéologique) ainsi que les raisons de la recherche d'une unité que nous retrouvons dans les luttes viticoles.

Le deuxième élément fondamental de cette étude tient à l'enracinement de l'enquêteur dans une collectivité rurale limitée mais bien connue. Cette situation a permis l'implication des intéressés eux-mêmes dans la recherche; une relation de collaboration véritable a pu s'établir avec le producteur de l'étude. Elle a permis d'analyser comment les impulsions qui viennent du système capitaliste sont reçues et intériorisées par l'économie paysanne, comment elle réagit à son environnement économique. Cet enracinement a permis à l'auteur d'analyser la crise au travers des rapports soiaux les plus fondamentaux dans la vie d'un viticulteur, de prendre en compte la crise telle qu'elle est vécue et ses répercussions directes, le but ultime étant la démonstration de la réalité de la crise viticole et la démystification de quelques mythes relatifs à la viticulture et aux viticulteurs du Midi.

Le plan lui-même veut faire ressortir ces préoccupations et ces convictions.

Un rapide survol historique permet d'analyser l'évolution de la viticulture languedocienne depuis 1907. Ce retour sur le passé se justifie car il permet une meilleure compréhension des phénomènes. Il est inséparable des événements actuels. Une communauté de problèmes posés à la région - crise structurelle de la viticulture évidemment, mais aussi faiblesse de l'emploi industriel (atteignant à peine la moitié de la movenne nationale), politique de grands équipements servant essentiellement au profit monopoliste, poids considérable du chômage et de l'exode des jeunes - ont fait du Languedoc un lieu où s'exprime la convergence des luttes, créant un sentiment de solidarité que traduit un mot d'ordre: "Volem viure al païs ».

C'est dans ce contexte qu'il faut placer le regain de sensibilité aux luttes du passé, telles que la "révolte des gueux" de 1907, et l'intérêt réel porté à une culture occitane, riche et variée, qui s'exprime à travers une floraison de chanteurs, d'écrivains, de troupes de théâtre... assurant la continuité des luttes d'hier à celles d'aujourd'hui, qui énoncent "mon païs escourchat $»$.

Les thèmes abordés par la suite recouvrent l'étude des rapports sociaux les plus fondamentaux dans la vie d'un viticulteur et donc les domaines dans lesquels la manifestation et les répercussions de la crise viticole sont 
vécus directement. C'est particulièrement sensible dans tout ce qui touche à la question foncière, notamment en ce qui concerne les facteurs de déblocage du marché foncier. La prédominance des exploitations familiales et de la petite propriété paysanne explique (cf. notion de patrimoine) le "gel " des structures foncières et du marché foncier, traduisant une amorce de concentration par glissement de catégorie. Par ailleurs, le caractère coûteux de l'investissement foncier, au moment où émergent de nouvelles exigences quant à l'augmentation des superficies cultivées, entraîne les viticulteurs à rechercher des solutions qui leur permettent d'éviter l'achat de la terre. C'est dans ce contexte que nous replacerons le développement récent des fermages et métayages dont certains comportent des dangers encore sous-estimés. Le Groupement foncier agricole est-il, par exemple, une solution possible pour éviter l'achat ?

L'étude du travail et des moyens de production, permet, au-delà de la mise en évidence des éléments de différenciation des exploitations, d'approcher, d'une part, les conséquences de la crise au niveau du quotidien des vitiulteurs (conditions de vie et de travail, conception du « métier »...) et, d'autre part, de mesurer l'écart qui se creuse entre l'intensification des efforts des travailleurs de la vigne et leurs difficultés de plus en plus grandes à survivre.

L'évolution des rendements met en évidence le choix économique effectué : quantité ou qualité ? L'étude de l'encépagement et des types de vins produits, nous permet de réfuter la thèse selon laquelle la crise viticole est engendrée par une production de mauvaise qualité. Nous sommes loin de l'affreuse "bibine" invendable en l'état et qui justifierait les importations massives de vins de forts degrés d'Italie, après ceux d'Algérie. Dans quel sens évoluent les efforts des producteurs, et en quoi en sont-ils récompensés. L'implantation de la monoculture de la vigne est-elle justifiée dans le Minervois? Les caractéristiques physiques et climatiques de cette région pourraient-elles permettre une nouvelle mise en valeur qui assurerait le maintien d'une population aussi dense et dispersée dans une multitude d'exploitations familiales. Y a-t-il une solution de substitution? La vigne fait vivre l'essentiel de la population, mais de plus en plus mal et la crise viticole a des répercussions directes sur le monde du travail. Le nombre des salariés diminue au profit d'un accroissement de la participation de la main-d'œuvre familiale, modifiant sensiblement les relations entre "entreprise agricole» et «ménage». L'idéologie de la propriété évolue; de timides répercussions se traduisent dans le développement des formes associatives.

Comment les viticulteurs ont-ils résolu leurs nouveaux problèmes de vinification et de commercialisation? La production de vin de qualité nécessite des investissements coûteux qui viennent augmenter les coûts de production mais qui ne se traduisent pas par une augmentation équivalente de leur rémunération. Ainsi il ne suffit pas de produire un bon vin, encore faut-il le vendre sans le brader... C'est renvoyer à l'organisation et à la concentration du négoce, ainsi qu'au "désordre " des marchés, qui entraînent des importations dont l'aberrante conséquence est l'envoi à la 
chaudière de vins de qualité. Face à la mutation du marché du vin, quelles sont les capacités de résistance et d'organisation des viticulteurs? La structure coopérative, qui a longtemps constitué un éran de défense de la petite exploitation, peut-elle continuer à jouer ce rôle ? Dans la crise, les faiblesses de l'appareil coopératif apparaissent, remettant en cause ses structures de fonctionnement et notamment les statuts qui l'ont créé. Coopérateurs et vinificateurs en cave particulière souffrent des mêmes faiblesses: manque de moyens financiers et structures commerciales incomplètes et fragiles. De tout ceci vient, en conséquence une baisse continue des revenus, qui engendre la paupérisation de l'ensemble des viticulteurs, mais qui met en évidence l'énorme capacité de résistance, dans la crise, de l'exploitation familiale. Les groupements à grande éhelle sont-ils une solution pour sortir de ces difficultés? Quels problèmes peuvent-ils résoudre ? La crise viticole recouvre-t-elle une situation de surproduction permanente? Et une baisse continue de la consommation?

Comment ont évolué importations et exportations? Et pourquoi un des premiers producteurs mondial du vin importe-t-il ? Il semble qu'il faille rechercher à un niveau économique international une des principales causes de l'aggravation de la crise viticole, dans l'organisation actuelle du marché commun. Qu'a apporté en fait ce marché de 220 millions de consommateurs qu'espéraient les viticulteurs du Midi, et quelles perspectives d'avenir engendre-t-il ?

Tout au long de ces développements, soulevant des questions fondamentales par rapport à la compréhension de la crise viticole, nous nous sommes efforcé de mettre en évidence les éléments de différenciation des viticulteurs, qui font que ce vignoble, loin d'être homogène, est composé de catégories qui disposent, dans la crise, de ressources différentes. S'il est vrai que la grande majorité des viticulteurs est touchée par la crise, ils la subissent à des degrés inégaux, engendrant des perspectives de reproduction sélectives.

Ce plan d'ensemble est à restituer par rapport à des considérations générales, qui résultent de la confrontation d'une expérience véclie et d'une démarche théorique.

Compte-tenu de ce que la "crise viticole " a donné lieu à de nombreuses analyses générales, nous avons choisi d'alimenter la réflexion à partir d'un matériau très concret : la commune de Laure-Minervois.

La méthode d'enquête et de travail correspond à la nécessité de coordonner données qualitatives et données quantitatives. Une première série d'entretiens auprès des viticulteurs et de techniciens et syndicalistes a permis de cerner les problèmes à analyser et de déterminer questions, thèmes d'études et champs d'investigations. Les données qualitatives furent recueillies par entretien direct. Les exploitations furent choisies comme unité d'observation et furent visitées durant l'année de travail. Les chefs d'exploitations, ainsi que certains ouvriers agricoles furent l'objet d'un entretien ouvert mais systématique dans une trentaine d'exploitations appartenant aux différentes catégories. Furent également l'objet d'enquêtes les responsables professionnels locaux et régionaux (techniciens et ingé- 
nieurs de la Chambre d'Agriculture, SUAD.) les représentants des caisses locales et régionales du Crédit Agricole, le fournisseur principal des carburants et produits de traitement et un gros concessionnaire en matériel agricole de Carcassonne. Quant aux responsables de la cave coopérative, nous pouvons parler d'une collaboration continue avec eux. Ainsi doit-on lire notre travail comme l'expression d'une démarche critique en même temps que comme un témoignage d'attachement filial.

M. P.-B.

\section{LES AVATARS DE L'INDUSTRIE RURALE DANS LE SUD-OUEST}

Devenues réserves d'espace après l'exode des dernières décennies, nos campagnes sont-elles restées des réservoirs de main-d'œuvre (2) ? Les zones rurales du Gers de l'Aveyron ou du Tarn-et-Garonnaise ont vu s'implanter, à l'écart des "grands axes de circulation » ou des "pôles d'attraction urbaine » des usines appartenant à des secteurs de pointe. Le critère d'isolement parait donc relatif : il est lié au niveau de développement des fores productives dont dépend en premier lieu la possibilité de fractionner les processus de production. Aujourd'hui, le milieu rural le plus "sous-développé » - c'est-à-dire le plus "paysannisé » - n'est plus seulement considéré comme un marché important; il est devenu un espace de production potentiel avec des qualités propres que les progrès les plus récents de la division du travail permettent désormais d'exploiter.

Dans le même temps des notions modernistes, la "qualité de la vie", etc., acompagnent la politique d'aménagement destiné à favoriser le redéploiement industriel, en lui donnant un contenu idéologique nouveau. Les agrariens de la III $^{\circ}$ République opposaient les vertus paysannes aux désordres des villes. Aujourd'hui, cette méfiance latente face aux sociétés urbaines et les dangers potentiels, sans cesse soulignés, des concentrations humaines, associées à des réalités plus proprement économiques provoquent un retour vers le milieu rural désormais considéré moins comme un conservatoire de valeurs sociales que comme un espace à prendre.

L'association du fait industriel - dans son sens dynamique - et du milieu rural est présentée comme une idée neuve située à l'origine d'une politique volontariste d'aménagement de tout le territoire, rompant avec les choix originels des pôles de développement. Pourtant, l'industrie fut très vivante dans les campagnes - véritable berceau de la révolution industrielle - dont l'histoire économique du dernier siècle fut celle d'une désindustrialisation continue, première responsable de l'exode. Notre démarche a donc consisté à considérer l'industrie rurale actuelle non comme un phénomène récent qui résulterait à la fois d'une volonté admi-

2. Jean-Louis Nembrini, Les avatars de l'industrie rurale dans le Sud-Ouest. Toulouse, 1978, Université de Toulouse-Le Mirail (Thèse de $3^{\circ}$ cycle, Géographie), 200 p., 9 fig., 15 tabl., biblio., annexes. 